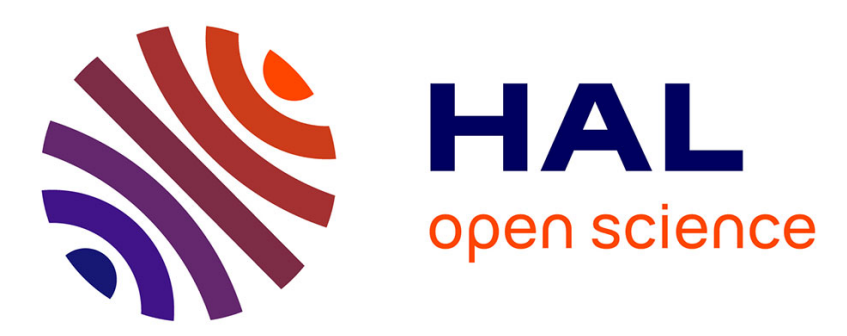

\title{
Affects, énonciation, et troubles de la parole dans la maladie d'Alzheimer
}

C. Hazif-Thomas, G. Chandès, Philippe Thomas

\section{To cite this version:}

C. Hazif-Thomas, G. Chandès, Philippe Thomas. Affects, énonciation, et troubles de la parole dans la maladie d'Alzheimer. NPG: Neurologie - Psychiatrie - Gériatrie, 2019, 19 (110), pp.105-109. 10.1016/j.npg.2018.10.002 . hal-02073901

\section{HAL Id: hal-02073901 \\ https://hal.science/hal-02073901}

Submitted on 22 Oct 2021

HAL is a multi-disciplinary open access archive for the deposit and dissemination of scientific research documents, whether they are published or not. The documents may come from teaching and research institutions in France or abroad, or from public or private research centers.
L'archive ouverte pluridisciplinaire HAL, est destinée au dépôt et à la diffusion de documents scientifiques de niveau recherche, publiés ou non, émanant des établissements d'enseignement et de recherche français ou étrangers, des laboratoires publics ou privés.

\section{(ㄷ)(1) $\$$}

Distributed under a Creative Commons Attribution - NonCommercial| 4.0 International 
Version of Record: https://www.sciencedirect.com/science/article/pii/S1627483018301570

Manuscript_ee149b5e5c6eaefb03e448355313e0e8

\title{
Affects, énonciation, et troubles de la parole dans la maladie d'Alzheimer
}

Affects, enunciation, and speech disorders in Alzheimer's disease

\author{
Cyril Hazif-Thomas ${ }^{1}$ \\ Gérard Chandès ${ }^{2}$ \\ Philippe Thomas ${ }^{3}$
}

1. Psychiatre et gériatre. Chef de service de psychiatrie du Sujet Âgé. (SPURBO, EA 7479). CHRU de Brest. Route de Ploudalmezeau, 29820 Bohars

2. Professeur de sémiotique

3. Psychiatre et gériatre. Chercheur associé

2 et 3 : Centre de Recherches Sémiotiques (CeReS, EA 3648) Université de Limoges. 39 rue Camille Guérin, 87000 Limoges, France.

Pas de conflit d'intérêts déclarés par les auteurs

Mail auteur 1 : cyril.hazifthomas@chu-brest.fr

Mail auteur 2 : gerard.chandes@unilim.fr

Mail auteur 3 : philippe.thomas.2008@orange.fr 


\section{Résumé}

La maladie d'Alzheimer ne se restreint pas à des désordres mnésiques. II s'y associe d'autres troubles cognitifs. Les troubles du langage ont un sévère retentissement sur la vie sociale des patients. L'appréhension du langage et des troubles du comportement est utile mais délicate d'interprétation dans sa pratique clinique auprès des personnes âgées démentes. Les liens unissant mémoire, langage et affects sont analysés afin d'une part de bien différencier trouble du langage et trouble de la parole et d'autre part de prendre en compte la prise en soin relationnel de la mémoire de vie de l'âgé. II importe à cet effet de favoriser l'assise énonciative de la personne soignée, levier utile de la pensée de secours face aux conséquences des troubles cognitifs, approche thérapeutique encore trop largement ignorée des soignants. Nous faisons dans cet article une revue des interrelations entre affects, énonciation et énoncés verbaux ou comportementaux dans cette maladie.

Mots-clés : Maladie d’Alzheimer ; Langage ; Enonciation ; Sémiose ; Démence ; Analogon

\section{Summary}

Alzheimer's disease is not restricted to memory disorders. Other cognitive disorders can be involved. Language disorders have a severe impact on the social life of patients. Assessment of language and behavioral disorders is useful but difficult to interpret in clinical practice among demented elderly people. The links between memory, language and affects are analyzed, firstly to differentiate between language and speech disorders and secondly to integrate attention to the elderly person's life memories. To this end, it is important to promote the enunciative abilities of the person receiving care, a useful lever for reinstating thought in the face of the consequences of cognitive disorders, an approach that is still largely unfamiliar to caregivers. In this article, we review the interrelationships between affects, enunciation, and verbal or behavioral statements in this disease.

Keywords: Alzheimer's disease; Language; Enunciation; Semiosis; Dementia; Analogon 
Parler avec un malade dément est tout sauf simple et souvent malaisé, ne serait-ce que parce que les soignants ne savent pas ou peu mettre en place les conditions d'un dialogue qui retiennent l'attention du malade. Plutôt que s'entretenir dans un espace interpersonnel partagé entre malade et soignants, évoluant dans un univers de sens commun, ils se contentent trop souvent d'émettre des jugements sur des comportements maladroits. L'incompréhension mutuelle est alors souvent élevée au rang de problèmes relationnels, au lieu d'être regardée comme une opportunité d'échanges favorables à la réassurance identitaire du malade. Nous dégagerons ici, à propos d'un bref cas clinique, quelques pistes de solution afin de promouvoir la production d'une signification efficace à travers une pratique désordonnée d'un malade, production toujours possible, même dans la démence. II faut repérer dans le sens de sa communication l'homologue du sens de la vie énoncée par une personne fragilisée par la maladie, une personne considérée d'abord comme un énonciateur digne d'intérêt.

\section{Définitions}

On appelle énonciation toute action produite par un énonciateur lorsqu'il produit un énoncé adressé à un destinataire ou énonciataire, message oral ou écrit, non-verbal ou comportemental, dans une situation déterminée (1). L'énonciation renvoie à la question de qui parle ou agit, pour qui, quand et où. L'énonciation module l'énoncé, le transforme et parfois en inverse le sens. Elle est une activité langagière intentionnelle, exercée par celui qui parle (2). Elle correspond au fonctionnement de la langue lors d'un acte individuel d'utilisation. Au niveau de l'énoncé (ce qui est dit ou fait), on retrouve les traces de l'émetteur du message, l'énonciateur. Si l'énonciation est énoncée, des marques dans l'énoncé renseignent sur les lieux, temps et actants engagés dans l'énonciation (l'instance d'énonciation). Lorsqu'elle est rapportée, par exemple en citant un auteur dans un texte, elle correspond au simulacre de la présence d'un tiers (3).

\section{L'énonciation dans la démence}

La démence sénile de type Alzheimer (DSTA) s'accompagne de désordres importants dans l'usage de la langue. Le discours des malades se distingue de celui des personnes âgées 
saines par l'altération des fluences verbales (4), une réduction significative de la diversité du vocabulaire et de l'informativité du discours, et enfin, par une réduction remarquable de la complexité syntaxique $(5,6)$. Les énoncés du malade sont très souvent altérés, d'autant plus que la maladie évolue, rendant la communication difficile avec lui (7). « Mais cette absence de sens de l'énoncé n'obère pas le sens implicite de l'activité d'énonciation " (8). L'énonciation persiste longtemps dans cette maladie, même si elle finit par s'altérer avec le temps. Pour Quadery et Védie (8), il est donc impropre de parler de troubles du langage dans la DSTA, et mieux vaudrait parler de troubles de la parole. Réduire le langage des malades à une production de signes et de mots désordonnés dans un énoncé maladroit, c'est le rendre inapte à toute communication (9). L'important dans la relation avec le dément est donc de s'attarder moins à ce que dit le malade que comment il le dit, ne pas s'attarder sur ce qu'il fait, mais comment il le fait.

L'énonciation orale, les comportements réalisés devant une personne, pour lever toutes les ambiguïtés de sens renvoient à des codes de référence inscrits dans l'énoncé ou dans le comportement, appartenant implicitement au contexte de l'échange entre l'énonciateur et l'énonciataire (Tableau 1). C'est à partir du contexte que des codes de référence permettent de construire le sens d'une communication, un entre-deux entre l'énonciateur et énonciataire, permettant à un sujet malade de s'inscrire dans le monde (10).

\section{La place de l'affect dans l'énonciation}

L'affect est une puissance d'affirmation, inséparable selon Deleuze et Guattari (11), du plan d'immanence où s'organise la signification. Le plan d'immanence permet de disposer et de rendre manipulable par la pensée les objets pertinents dans l'espace perçu à un moment donné. L'affect remodèle en permanence la construction de ce plan, toujours instable, toujours agencé et réagencé par des affects-passions et des affects-actions, en permanence recomposé (12). La subjectivité et la jouissance de ses désirs se manifestent essentiellement dans le discours. " C'est dans l'instance de discours où 'je' désigne le locuteur, que celui-ci s'énonce comme 'sujet'. II est donc vrai, à la lettre, que le fondement de la subjectivité est dans l'exercice de la langue. Si l'on veut bien y réfléchir, on verra qu'il n'y a pas d'autre témoignage objectif de l'identité du sujet que celui qu'il donne ainsi lui-même sur lui-même» 
(13). Le 'je' du discours dans un texte écrit renvoie le plus souvent et directement au locuteur, mais pas exclusivement, dans une énonciation énoncée, un personnage peut être présenté et intégré au discours. Dans le discours oral, cette présence du 'je' qui autodésigne le locuteur, celui qui parle à quelqu'un, est encore plus marquée, et plus implicite pour celui qui l'écoute.

\section{Sujet de l'énonciation et démence}

Le sujet de l'énonciation, le 'je' du discours ne reflète pas cependant toujours la personne parlante dans la psychose ou parfois dans la démence. Dans ce cas, consciemment ou non, intentionnellement ou non, le 'je' est un grand Autre (14), qui se substitue et impose le discours et parfois sa volonté au locuteur, rendant l'énoncé du discours confus, générant de la perplexité chez l'énonciataire. Ce grand Autre qui entre en jeu perturbe donc l'énonciation, mais fluctue d'un moment à l'autre dans le discours du malade (14). Le psychotique peut se sentir prisonnier et possédé par un tiers qui lui impose sa volonté, son discours et ses comportements. Le psychotique n'a pas accès à son désir, et « ce dont parle toute énonciation, c'est du désir " (15). Dans la démence, le malade a longtemps accès à ses désirs, du moins lorsque la maladie est au stade évolué et modéré, mais de façon plus ou moins élaborée, en raison des difficultés cognitives et tensives auxquelles il se confronte. Son discours peut être alors chaotique. Lorsque la maladie est très évoluée, l'accès au désir est compromis, et une dérive psychotique est fréquente, avec des manifestations délirantes et hallucinatoires.

\section{Désir et énonciation}

Contrairement au sujet névrosé, la personne psychotique ou profondément démente est en dehors du circuit du désir, pouvant parler (PARL) mais ces paroles peuvent rester sans signification propre, sans agencement symbolique à partir de son être singulier (ÊTRE), ce que Lacan (16) désignait comme PARLETRE. Il est un sujet parlé et non plus parlant. Dans la démence comme psychose, l'énonciation psychotique met en cause le sujet de l'énonciation, un grand Autre pouvant s'y substituer. De plus la maladie perturbe l'énoncé, sans organisation symbolique puisque non ancrée dans le désir, et donc sans structuration 
logique, sans finalisation du contenu, excluant donc un interlocuteur éventuel, un énonciataire de son discours. L'énonciataire ne se reconnaît pas comme personne à qui le discours s'adresse ou qui est concerné par les actes du malade, ou simplement ne cherche pas à le comprendre (7).

\section{Cas clinique}

Mr H 82 ans est un ancien journaliste, éditorialiste dans un grand journal national. II vit en EHPAD, en raison des conséquences d'une démence de type Alzheimer évoluée (dernier MMSE : 12/30).

Son épouse rapporte, à domicile, ces dernières années, une longue histoire personnelle d'attitudes ritualisées autour de l'écriture (décousue) d'éditoriaux attendus soi-disant par la rédaction du journal. N'importe où dans la maison, à n'importe quelle heure, sur n'importe quel papier, Mr H écrivait. C'était là l'origine de multiples disputes à ce propos avec elle, disputes qui continuent à l'EHPAD avec les soignants, car $\mathrm{Mr} \mathrm{H}$ a continué d'agir ainsi. Dépressif, triste, $\mathrm{Mr} \mathrm{H}$ est conscient de sa maladie « $\mathrm{Mr} \mathrm{H}, \mathrm{Mr} \mathrm{H} \ldots$,., il n'est plus rien $\mathrm{Mr} \mathrm{H}$ ».

Il faisait beau ce jour-là et, en tenue estivale, monsieur $\mathrm{H}$ s'est installé dans un banc du jardin (Figure 1). Dans sa confusion, il a saisi sur une table, ce qui ressemblait vaguement à un crayon, une petite cuillère et commence à écrire sur un calepin qui trainait là. II écrit de façon appliquée depuis un long moment déjà quand une aide-soignante lui fait une remarque : «Monsieur $\mathrm{H}$, vous utilisez une cuillère au lieu d'un crayon !» La réponse de Mr $\mathrm{H}$ est disproportionnée et quelque peu agressive : «Occupez-vous de vos affaires. Monsieur $\mathrm{H}$, il travaille. »

\section{Discussion}

L'énonciateur, $\mathrm{Mr} \mathrm{H}$, comme sorti de son discours, n'entretient plus de rapport avec son locuteur (épouse, soignant) (17). Dans la psychose, comme dans la démence très évoluée ou dans l'autisme, l'individu qui parle en introduisant un 'il' (Iléité, dysharmonie évolutive lorsque I'on parle de la clinique de l'enfant)(18), un autre, "des voix ", n'assume pas son discours en tant que sujet, il n'est plus nécessairement l'instance qui prend en charge 
l'énonciation, qui se donne pour responsable de l'énoncé. Par exemple dans son délire, un patient psychotique qui entend des voix qui le commandent ne se sent pas forcément responsable de ses actes et de ses dires. Pour Mainguenaud (19), l'énonciation est alors soutenue par aucun sujet réel. Nous voyons à propos de Monsieur $\mathrm{H}$, qu'il parle de lui-même en se désignant comme un autre, un 'il'. Monsieur $\mathrm{H}$ : « $\mathrm{Mr} \mathrm{H}, \mathrm{Mr} \mathrm{H}$, il n'est plus rien, $\mathrm{Mr} \mathrm{H}$. "Ou encore "Occupez-vous de vos affaires, $\mathrm{Mr} \mathrm{H}$, il travaille », rejetant ici une tentative de manifestation d'un locuteur, un soignant, qui observe ses activités. Monsieur H n'est pas au stade de la disparition de tout désir, sa démence n'est pas suffisamment infléchie en ce sens; il assume, en revanche, avec beaucoup de persévérance sa perte d'identité et il cherche à la restaurer.

L'absence d'accès ou l'accès partiel au désir, donc au préconscient, ne permet pas l'élaboration d'une pensée symbolique et se traduit par un énoncé chaotique. $\mathrm{Mr} \mathrm{H}$ bâtit son propre plan d'immanence, un pare-feu dans le chaos envahissant, en créant un concept supplétif, un analogon. Pour Deleuze et Guattari (11), "le chaos n'est pas seulement le hasard. II n'est pas seulement une absence de déterminations " mais aussi, leur caractère éphémère, "la vitesse infinie à laquelle elles s'ébauchent et s'évanouissent ". Le chaos rend impossible la construction de relations entre les sémiotiques-objets et les rapports entre les concepts et, par conséquent, conduit à une incessante dissolution de toute construction signifiante de la pensée. La construction délirante de l'énoncé ou la production hallucinatoire, chez un psychotique, ou dans la démence évoluée, sont le témoin de la menace de la fragmentation du Moi. La conscience de la disparition du sujet de l'énonciation et de tout désir renvoie à une angoisse particulière, différente de l'angoisse de castration, l'angoisse de néantisation (20). Un malade dément comme un malade psychotique dans ses expressions et ses comportements maladroits luttent désespérément pour empêcher ou élaborer cette angoisse. Ne pouvant y arriver de façon satisfaisante seul, malgré ses maladresses et ce qui paraît à l'observateur extérieur être un comportement transgressif, il produit un plan d'immanence qui n'en est pas moins un appel au secours, répétitivement répété et en échec d'être compris de longue date (21). Rappelons que pour Deleuze et Guattari, « Le plan d'immanence n'est pas un concept pensé ni pensable, mais l'image de la pensée, l'image qu'elle se donne de ce que signifie penser, faire usage de la pensée, s'orienter dans la pensée... » (11). 
Dans la psychose, le sujet de l'énonciation, le grand Autre tout-puissant, non entravé, n'est pas assimilable comme dans la névrose à l'inconscient, il est totalement extérieur au sujet et n'entretient aucun lien symbolique avec lui (17). Dans la démence, ce grand Autre représente un reste de puissance, traduit par un 'il', ici chez $\mathrm{Mr} \mathrm{H}$, un 'je' dépersonnalisé, mais cependant un ' $j e$ ' encore capable de dire et de faire quelque chose qui garde du sens pour lui, et renvoie à des désirs inscrits dans le passé de $\mathrm{Mr} \mathrm{H}$. II garde donc un certain lien avec le malade. Le sujet, le 'il', qui parle par la bouche de Monsieur $\mathrm{H}$, partage encore avec lui les traits d'un 'je' véritable, chaotique, mais désirant, intentionnel tourné sur ce qui est encore possible. II "chaobiaise » (Chaos- biaise le désir, néologisme traduisant l'instantanéité de leur conjonction en les réunissant dans un même mot) en quelque sorte son approche symbolique.

La cuillère est un analogon de crayon, trace visuelle d'un début de deuil d'une réalité devenue inabordable. La persévérance de $\mathrm{Mr} \mathrm{H}$, son refus d'accommoder ses gestes vers une pratique normalisée témoignent de l'implication de $\mathrm{Mr} \mathrm{H}$ dans sa lutte contre une dépression conative (22) et non nécessairement vers la réussite d'un acte matériel. Il assume avec force une démarche pour se retrouver lui-même dans une pratique, dans une forme de remémorisation en acte, où il retrouve du bien-être et son identité menacée par la maladie (21). Monsieur $\mathrm{H}$ montre sa conviction pour restaurer une fonction énonciative, pour réinstaurer un ' $j e$ ' énonciatif qui lui permettrait de se retrouver dans un jeu de langage signifiant, d'abord pour lui et probablement pour les autres. La " cuillère analogon » renforce le lien qui l'inscrit dans une nouvelle réalité singulière, intime, traduisible par le discours ou un acte porteur de sens pour lui. Monsieur $\mathrm{H}$ se réinscrit comme le véritable sujet de l'énonciation, dans la dignité d'une personne pouvant vivre dans un champ de présence où il retrouve identité et bien-être. La présence d'un tiers écoutant, contenant, bienveillant aurait pu valider par une parole adaptée la pratique de Monsieur H. En pointant le symptôme, en soulignant la maladresse transgressive de $\mathrm{Mr} \mathrm{H}$, le soignant renforce sa propre exclusion de l'énonciation de Monsieur $\mathrm{H}$. Il pointe certes le chaos mais il oublie le désir, il se condamne à un dérèglement sémantique, ne partageant aucun code commun avec le malade, il se démentifie lui-même (8). Le plan d'immanence du soignant et celui du malade coexistent, se superposent mais ne communiquent pas. Sans chercher à transmettre autre chose que ses normes, sans prendre la dimension affective et passionnelle sous- 
jacente assumée par $\mathrm{Mr} \mathrm{H}$ dans sa pratique, le soignant plaque son plan sur celui de $\mathrm{Mr} \mathrm{H}$. II disqualifie et ruine alors les efforts de $\mathrm{Mr} \mathrm{H}$ pour se réinscrire comme sujet véritable de son énonciation. Au contraire, en validant ce que fait $\mathrm{Mr} \mathrm{H}$, il conforterait la prise de position subjective du malade dans sa pratique, il s'instaurerait lui-même, de fait, énonciataire, restaurant ainsi la totalité de l'assise énonciative. II offrirait au malade la possibilité d'avoir un tiers à s'adresser, et partant, il faciliterait la reprise d'une certaine cohérence langagière, la situation du dialogue étant expressément déterminée et garantie. Ainsi il offrirait l'opportunité d'une pensée de secours, une possibilité de suppléer la loi symbolique défaillante $(17,23)$, s'offrant comme Moi auxiliaire à l'aide duquel le patient pourrait, le temps d'un échange intersubjectif, s'extraire de la démence.

\section{Conclusion}

Faciliter la pensée de secours implique, pour nous soignants, d'écouter la parole et non seulement le langage des malades, de prendre le risque d'investir l'aspect analogique de la pensée, d'adopter une démarche systémique et de sortir de l'exclusivité de l'approche cartésienne trop cartésienne, purement digitale, survalorisée aujourd'hui, c'est-à-dire, pour notre propos, restreinte au seul traitement cognitif de l'information. La pensée humaine est bien plus riche de consonnances affectives, d'interrelations complexes que de composantes rationnelles. Elle est forte de vécu fantasmatique et d'attendus motivationnels qui convoquent la prise en compte de l'énonciation des patients, autrement déshabités de leur singularité langagière. Apprendre à « chaobiaiser » de concert avec les patients déments est aussi utile que de s'intéresser aux biais cognitifs qui orientent leurs jugements, et par-delà, leur discours et leur comportement. C'est à ces conditions que les soignants peuvent dresser un plan de soins personnalisés, non plaqué sur leur norme, plan superposé et compatible avec le plan d'immanence construit par le malade pour sa gestion du chaos démentiel. 


\section{Références}

1. Benveniste E. L'appareil formel de l'énonciation. Langages 1970; 17: 12-8.

2. Anscombre J-C, Ducrot O. L'argumentation dans la langue. Langages 1976; 42: 5-27.

3. Greimas AJ, Courtés J. Sémiotique. Dictionnaire raisonné de la théorie du langage. Hachette, Paris, 1979: 125-8.

4. Hazif-Thomas C, Billon R, Thomas P. Narrativity and memory analysis in Alzheimer's disease. Op J Psych 2017; 7: 107-307.

5. Hyeran L. Langage et maladie d'Alzheimer : analyse multidimensionnelle d'un discours pathologique. Thèse de doctorat en Sciences du langage, Montpellier, 2012. http://www.theses.fr/2012MON30059 Consulté le 1 octobre 2018.

6. Wirotius J-M, Pétrissant J-L. Langage et discours dans la démence. J Readapt Med 2005; 25(2): 94-8.

7. Hazif Thomas C, Thomas $\mathrm{P}$, Chantoin S. Langage, Motivation et oubli dans la maladie d'Alzheimer. Alzheim Actual 1999; 11-12(147): 6-12.

8. Quaderi A, Védie C. Néologisme et maladie d'Alzheimer. Ann Med Psychol 2007; 16: 680-4.

9. Irigaray L. Le langage des déments. Editions Mouton, Paris, 1973.

10. Silverstein M. Indexical order and the dialectics of sociolinguistic life. Language Comm 2003; 23: 193-229.

11. Deleuze G, Guattari F. Qu'est-ce que la philosophie ? Les Éditions de Minuit (coll « Critique »), Paris, 1991.

12. Delourme C, Lecercle J-J. "Affect ", in Le vocabulaire de Gilles Deleuze (sous la dir. Robert Sasso et Arnaud Villani). Les Cahiers de Noesis 2003; 3: 32-3.

13. Benveniste $E$. De la sujectivité dans le langage In: Problèmes de linguistique générale. Tome 2. Editions Gallimard, Paris, 1966.

14. Lacan J. Le Séminaire II. Le moi dans la théorie de Freud et dans la technique de la psychanalyse. Editions du Seuil, Paris, 1977 [1966].

15. Lacan J. Le Séminaire XI. Les Quatre Concepts fondamentaux de la psychanalyse. Editions du Seuil, Paris, 1973 [1964]: 129.

16. Lacan J. Le Séminaire III. Les Psychoses. Editions du Seuil, Paris, 1981: 285.

17. Samacher J-Y. L'énonciation dans la psychose. Psychol Clin 2013; 36: 157-72.

18. Grollier M. Analyse d'énoncés d'enfants autistes à partir de la psychanalyse, quelle ouverture pour une énonciation. Evol Psychiatr 2007; 72: 421-35.

19. Maingueneau D. Les termes clés de l'analyse du discours? Collection "Points et essais". Editions du Seuil, Paris, 2009.

20. Jones E. Early development of female sexuality. Internat J Psycho-Analysis 1927; VIII: 459-

72.

21. Thomas $P$, Chandès $G$, Hazif Thomas $C$, et al. Analyse du sens d'un trouble du comportement dans la démence. Ann Med Psychol 2017: 906-13.

22. Hazif-Thomas $C$, Reber G, Bonvalot $T$, et al. Syndrome dysexécutif et dépression tardive. Ann Med Psychol 2005; 163: 569-76.

23. Ploton L. À propos de la maladie d'Alzheimer. Gerontol Societ 2009; 32: 89-115. 
Tableau 1 : Exemple de transformations du sens entre énoncé et énonciation lors du dialogue d'un malade dément avec un soignant dans un établissement. Référencement à des codes communs.

\begin{tabular}{|c|c|c|c|}
\hline & $\begin{array}{l}\text { Ce qui est énoncé } \\
\text { (Wording) }\end{array}$ & & $\begin{array}{l}\text { Ce qui est compris } \\
\text { grâce à l'énonciation } \\
\text { (Statement) }\end{array}$ \\
\hline Malade & $\begin{array}{l}\text {-J'ai faim } \\
\text { Affirmation d'un } \\
\text { état }\end{array}$ & Soignant & $\begin{array}{l}\text { Mr H pense qu'il est } \\
\text { I'heure de manger }\end{array}$ \\
\hline Soignant & $\begin{array}{l}\text {-Il est 10h du matin } \\
\text { Rappel de l'heure }\end{array}$ & Malade & $\begin{array}{l}\text { Ce n'est pas encore } \\
\text { l'heure du déjeuner }\end{array}$ \\
\hline Malade & $\begin{array}{l}\text {-Ah, bon. Merci } \\
\text { Prend acte de } \\
\text { l'heure et remercie } \\
\text { pour l'information }\end{array}$ & Soignant & $\begin{array}{l}\text { Mr H a compris et va } \\
\text { attendre midi }\end{array}$ \\
\hline
\end{tabular}




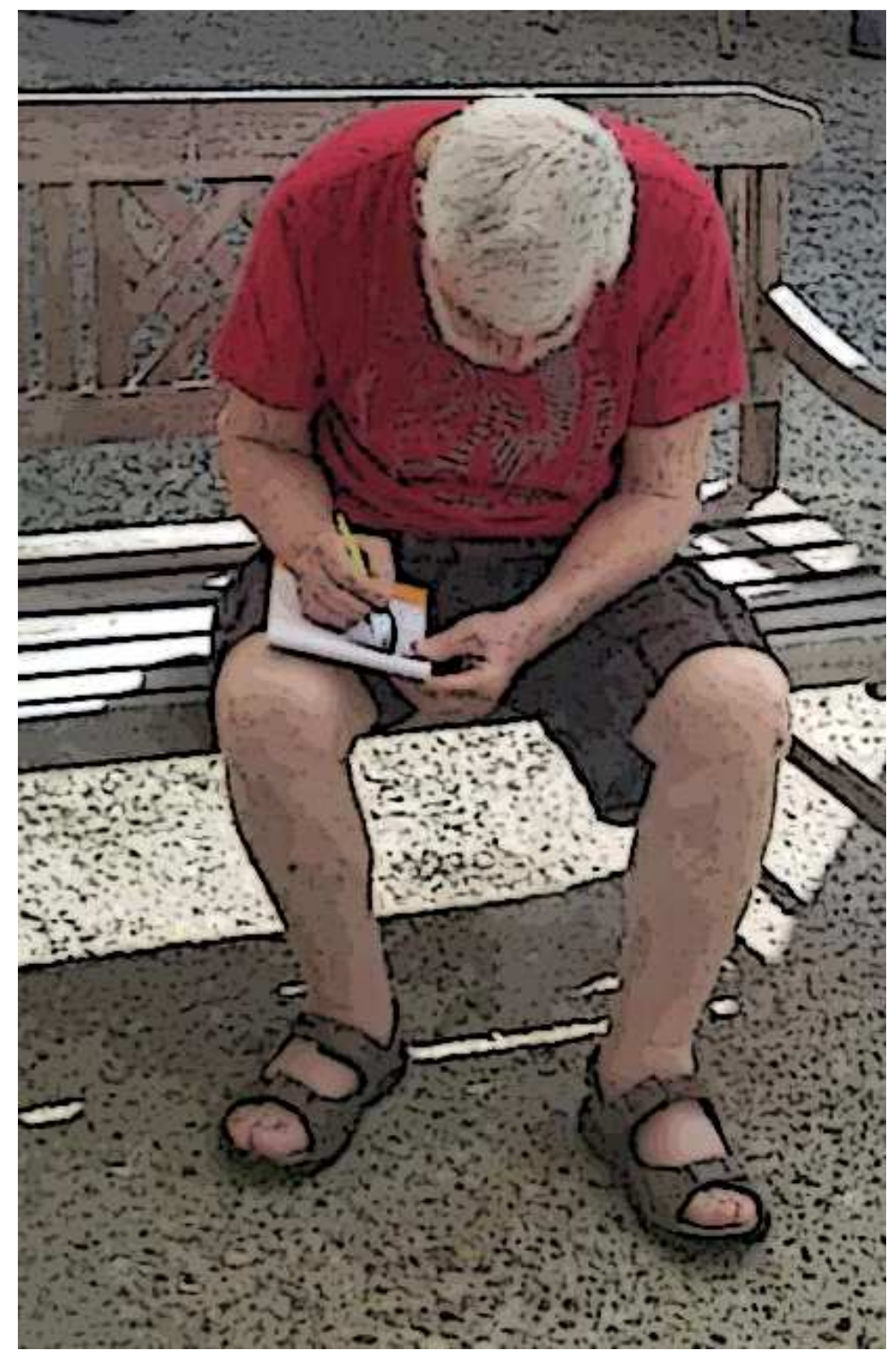

Figure 1 : Mr H écrivant avec une cuillère. 